\title{
Virtual Environments and the Future of Human- Computer Interfaces: The Electronic Frontier in Social Context
}

\author{
Ralph Schroeder \\ Department of Human Sciences \\ Brunel University, Uxbridge \\ Middlesex UB8 $3 P H$ \\ United Kingdom \\ Telephone: 44 (0)895 274000 \\ E-mail:Ralph.Schroeder@brunel.ac.uk
}

\section{Synopsis}

Virtual environments have assumed a prominent place among the possibilities for the human-computer interfaces of tomorrow. Yet the simultaneous transformation of a number of information and communication technologies makes it difficult to assess how computergenerated simulated worlds will become part of interface design. This paper argues that thinking about the potential role of virtual environments requires locating them among a range of related technologies for interacting with information and communication tools on the one hand - and identifying the dynamics of the social contexts in which they operate on the other. The relation between these two levels, in turn, can provide us with a conceptual framework for identifying the distinctive contribution that virtual environments can make to humancomputer interface design.

Keywords:

virtual environments, human-computer interfaces, electronic media, sociology of computing. 


\section{Introduction}

Virtual environments, computer-generated worlds in which users experience a sense of presence, are rapidly becoming an important mode of human-computer interaction. But they are merely part of a wider transformation which consists of the convergence among a number of information and communication technologies, such computers, television and computer-mediated communication.

This essay will explore this electronic frontier by examining some of the social settings in which virtual environments or virtual reality systems are being used. It will be argued here that in order to make sense of how virtual environments may contribute to the future of these new modes of human-computer interaction, some important clues can be gained through analysis of the forms of social action and interaction within these environments and of the responses of users to existing systems. And although it may be impossible to foresee the humancomputer interfaces and the virtual environments of the future, it is important to think about the various possibilities that may lie ahead, if only because this may help us to understand the factors that will shape these new tools.

\section{Virtual Reality and the Limits of Simulation}

Virtual reality technology was initially thought of as a way of eliminating the interface. In Ivan Sutherland's 'Ultimate Display', the idea was that the user could interact directly, by means of the senses, with the world generated by the computer display (Sutherland, 1965). The simulated world would be so vivid that the user would experience it as real. Walker has recently restated this aim: Virtual environments 'will begin to break down the barrier between the user and the world inside the computer (1990: 447). Given the existing state of the technology, however, it is difficult to say to what extent virtual reality has embarked on this process.

In order to think about the aim of eliminating the interface, it may 
be useful to take these ideas to their logical conclusion - and then work our way backwards again. For this purpose, we shall need a thought-experiment: Let us assume that it is possible to create a completely realistic computer-generated world with which users can interact. What would this virtual world look and feel like? It would still consist of human beings, using whatever input/output channels they chose or had available to them, to interact with a simulated environment, possibly including other users. That is to say, no matter how 'natural' the interface or how 'realistic' the environment, users will still be aware that they are making use of a tool.

This point can be clarified by considering what might be entailed by asserting the opposite viewpoint, namely, that in the end, users would be unable to distinguish between a virtual environment and the real, physical world. This idea, which has fuelled much of the speculation surrounding virtual reality, can be discounted, since it rests on denying that there is a distinction between the physical and simulated worlds. Yet such a conflation would only be called for if it were to be the case that human behaviour could in some way become so profoundly altered through the extensive use of future information and communication technologies, so that this altered behaviour would itself affect our perception of reality. But this is an analytically separate issue which is not only related to virtual reality, but the way in which human perception and behaviour are affected by information and communication technologies generally. And as far as this general issue is concerned, it is difficult, as Biocca has pointed out, to make reliable pronouncements even in the case of technologies that have been studied extensively, such as television (1992: 14-15). How much more so, then, in the case of virtual reality, a technology about which as yet little is known in this regard?

It is true, of course, that the degree to which users are aware of using a tool will vary. That is why the sense of 'presence' or 'immersion' in virtual environments is currently the subject of extensive debate among virtual reality researchers (for an overview of some of these 
issues, see Schroeder, 1994). But howsoever 'presence' or 'immersion' may come to be defined, the machines which make 'presence' and interaction possible will still need to be subject to the rules of how humans perceive simulated worlds, and this, as I hope to show, depends on the uses to which they are put. The aim of virtual environments is therefore not so much 'realism', but rather creating virtual reality systems or machines which suit different needs for manipulating simulated worlds.

If the point that virtual environments must, in the end, serve human needs, seems an obvious one, it is nevertheless important to be clear about this in the light of misleading ideas that have developed around this technology. Consider, for example, the notion that virtual reality will allow us to create worlds in which it will be possible to eliminate social ills such as disease, or the suggestion that interpersonal relations will become profoundly altered with prolonged exposure to virtual reality. Both possibilities have been put forward without, it seems, taking into consideration that virtual environments are simulations and that multi-user virtual worlds are, in effect, nothing more than using a telephone with additional input and output channels apart from audio. If all this is so, we must keep our feet firmly planted on the ground and think, not about eliminating disease and creating new kinds of interpersonal relationships, but about how the new tools may apply to specific settings and needs.

There is a parallel here with artificial intelligence. As Collins has argued, any computer that does the work of human thinking cannot but take social behaviour into account. Indeed, it will have to have emotions and engage in a variety of social relations if it is to succeed. This is because the limits on computer models so far have resulted from the fact that they represent intelligence as if it were a freestanding individual mind', whereas human intelligence is in fact both emotional and social (Collins, 1992: 156). A similar point could be made in relation to virtual reality (and in fact, it has, inasmuch as it has been noted that the use of the word 'reality' here is, strictly speaking, 
mistaken). That is, virtual environments should not aim to reproduce reality itself as if it were independent of users, but rather to create environments that reflect particular human and social requirements.

\section{Virtual Environments and Social Contexts: The Example of Entertainment Games}

At this point we can work our way back to the present. Whatever the current convergences and developments among information and communication technologies may bring, the technologies of humancomputer interaction and of virtual environments will have to accommodate the specific uses to which they are put. They cannot aim to be anything other than what they are; namely, tools for interacting with simulated worlds. Thus there is a need, in the case of the humancomputer interface, to allow information to be manipulated in a way that suits the user, and in the case of virtual environments, to meet the users' needs in engaging with environments.

To illustrate this point, we can take the examples of two VR entertainment games, the $\mathrm{W}$ Industries game 'Legend Quest' and Kinney Aero's 'Fightertown' aircraft simulation. Focusing on entertainment games has the advantage that these are currently the most widely used virtual reality systems. That is, they are not just pilot projects confined to research centres. This is important in the light of a point that will be made about both games, namely that the way they are used depends importantly on the familiarity of users with similar games or skills-based activities outside the virtual worlds. Here it may also noted that if entertainment games seem to be a trivial example, similar considerations will apply to other uses of virtual environments, such as education (Schroeder, 1993). Not only this, but the most common early uses of new technologies (in this case, VR) often have a decisive influence on other applications.

If we take, first then, the experience of $\mathrm{W}$ Industries' 'Legend Quest'. This game is played with a head-mounted display and a hand- 
held joystick (for a brief account of the technical features, see Kalawsky, 1993: 39-40). The player, who takes on the role of a particular character, such as a dwarf or wizard, enters a virtual world for a brief period, typically between 5 and 20 minutes: Once 'inside' the virtual world, the game itself consists of a number of interconnected rooms in which a series of tasks, such as killing skeletons, finding objects and using keys to enter new rooms, are pursued. The format, in other words, is akin to a dungeons-and-dragons game, in this case set in an immersive virtual world which can be shared by up to four players.

There are many features of this game which deserve comment and which shed interesting light on the nature of virtual reality. What needs to be highlighted here (and this applies to other task-oriented VR games) is that the main feature of interaction in this case is the need to become accustomed to the environment. This is partly a matter of time. If players were able to spend a lot of time in this environment, then they might learn or simply get used to the idea of, say, orienting themselves in the rooms of this world by means of the joystick, or to manipulating virtual objects.

It might be objected that this is mainly to do with the fact that this is an entertainment game which deliberately limits the amount of time available and makes the tasks difficult. Yet this feature, I would argue, applies to immersive environments generally. When users experience immersive virtual reality systems for the first time, the success or enjoyment they derive from this depends on their ability to navigate within the virtual environment, rather than, say, on being impressed with how 'realistic' they find the virtual world. This observation has, for example, also been made in relation to other, non-entertainment game first-time uses of virtual reality systems (Bryan Cleal, personal communication, 6.4.1993). Even if then, for the sake of argument, we assume that users are able to spend as much time as they like in the virtual world, the need to orient themselves within the environment and control its elements is therefore likely to remain paramount. 
Let us turn to the second virtual reality gaming experience, the 'Fightertown' game (further details can be found in Giles, Schroeder and Cleal, 1994). This is essentially an aircraft battle simulation. Users sit in a very realistic model of a cockpit, complete with controls, and fly missions against other players while being briefed from a control tower. The display in this case is a cinema-like wide screen approximately twenty feet in front of the cockpit featuring highresolution computer graphics. This kind of simulation has also been called second-person virtual reality since the user is not within the environment itself, but rather in the cockpit cabin. The experience of this game is nevertheless closely akin to that of a virtual environment. The content of the game is highly absorbing in this case because of the complexity of the skills involved. Manipulating the controls in this case makes constant demands on the user's concentration, as it does in the case of flying a real aircraft. Even if, during the course of a game which lasts half an hour, the player becomes a skilled pilot, the challenge of battle with other players makes additional demands on the user's skills.

The important point about these two experiences of virtual environments is that the nature of interaction is very different. In the case of 'Legend Quest', interaction depends on the user's familiarity with the conventions of this type of game on the one hand, and on adapting to the skills requirements of orientation and navigation in a multi-levelled virtual environment on the other. It depends, in other words, on role-play and exploring the rules governing the environment. 'Fightertown', by contrast, requires the simultaneous exercise of a number of coordinated skills. Mastery of the controls of the aircraft demands an intense concentration of a rather technical kind. If we add the multi-user aspect of both games, which consists of adaptation to co-ordination in another environment in Legend Quest', as against tactical team play in 'Fightertown', it can be seen that the settings provide very different experiences of interacting with virtual environments.

This last comment about the way in which the multi-user nature of 
the virtual environment adds a further dimension to the differences between the experiences of 'presence' and interaction can be underlined by making a brief contrast between two further current uses of multiuser or shared virtual environments: between text-based virtual realities (Curtis and Nichols, 1993) as against primarily visual and aural shared virtual realities (apart from entertainment games).

The problems with existing text-based virtual realities, as Curtis (personal communication, 6.8.1993) has noted, are very much those that might be expected when the norms governing face-to-face interaction become suspended. Forms of anti-social or 'deviant' behaviour assert themselves, behaviours that are less possible in faceto-face interaction. Hence solutions, such as censorship, need to be found. Alternatively, new rules of interaction, such as the new forms of 'speech' to indicate mood (the smiling sideways face :-) is the most commonly cited example) are becoming widespread in computermediated communication.

The problems facing visual and aural virtual realities are just as complex. How, for example, should users be represented to each other? Do they need 'realistic' bodies (see Slater and Usoh, 1993), or is iconic representation, say, in the shape of a rectangle with cartoon-like facial features, sufficient in a three-dimensional environment (Fahlen, 1993: 83)? In this case, too, different solutions will emerge, depending on the requirements of users. What is interesting here is that having a realistic 'virtual' body may not necessarily enhance the users' sense of presence and interaction. Instead, this will be dependent on the tasks or the role of users in the virtual environment.

These are some of the features of shared virtual realities. Most virtual reality systems that are currently in use, however, are for single users. Nevertheless, the issue, and one that has long been recognized in the case of the design of graphical user interfaces (for example, Salmon and Slater, 1987: 474-5) is that the user's model of the world needs to be incorporated into the information environment which the user has to manipulate. But in the case of virtual environments, this means that the 
mode of interaction plays a crucial role in designing the system to meet the user's needs. If so, then Collins' suggestions about incorporating human interaction into artificial intelligence, or the suggestion that has been made here to take into account the settings in which these systems are used, apply just as well to single-user virtual environments.

\section{Human-Computer Interfaces, Virtual Environments and Media Wars}

Having examined some specific settings in which virtual reality is used, we are now in a position to examine the larger context in which these systems are being developed. One of the reasons why there has been so much uncertainty about the direction of virtual reality development is that a transformation among a number of information and communication technologies is currently under way. These include telephony and television, computing, computer-mediated communication, the digitization of sound and images, and broadband networks. This change is partly indicated by the use of new keywords in connection with these technologies, such as multimedia, the information superhighway, and interactivity.

Little is known about the shape of this transformation, apart from the likelihood of some kind of convergence among these devices and technologies. It is clear, however, that any aspect of this transformation will have an important influence on virtual environments. Two questions will be decisive here: One is whether virtual worlds will primarily take the form of single-user or of multi-user (or shared) worlds. The second concerns the nature of the virtual world itself, its level of complexity or 'world-likeness'. Unfortunately, the two cannot be divorced, and neither can they be separated from the larger framework of converging technologies in which they are embedded.

Regardless of the number of users in a virtual world, however, the representation of real-life processes in virtual environments can be enhanced by incorporating within them the simulation of the 
behaviours of environments and of humans. This is where simulation in the more narrow sense comes in because of the possibility of mutual benefit from the traffic between virtual reality and the more wellestablished fields of simulation and gaming. Simulation in this sense, by contrast with virtual reality, typically entails the translation of real-life behaviours and processes into symbolic notation, although definitions vary and sometimes include physical models of the world (Paul and Balmer, 1993: 1-3). This is important because virtual environments are not simply static but also comprise dynamic features, such as changes in the scenery. Other possibilities currently being explored include intelligent creatures (see Waldern, 1994). Or again, simulated social institutions, such as those of the SIMCITY interactive software programme (White, 1992), might also be incorporated within virtual environments. Although this avenue is not, to my knowledge, currently being pursued, this is perhaps only a matter of time.

To give just one illustration of the possibilities of this overlap between simulation in this sense and virtual reality, we can use the application of building safety. For example, the spread of fire can be modelled using three-dimensional computer graphics in relation to the simulated behaviour of human beings (again, represented threedimensionally) trying to escape an enclosed space. This combination of virtual reality and simulation modelling has been undertaken by the Colt International engineering firm (press release, 1.2.1994) using a desktop virtual reality system, that is, one which consists of a desktop computer display and 'spaceball' input device (in this case the Dimension Superscape system, briefly described by Kalawsky, 1993: 207). Much can be learned in this way about the relation between the parameters of the physical environment that has been modelled and patterns of human behaviour in relation to it. And again, it needs to be emphasized that this is only one among a range of projects that are currently in the process of bringing the simulation of behaviours and virtual reality systems closer together.

A system which incorporates patterned human behaviour in this 
way could, in the case of an immersive virtual world with which users can interact, create the impression of real-life processes. And although the possibility may at present seem remote, it can be envisaged that at this stage, and with much more complex environments and simulations of human behaviour, the argument made earlier about the limits of simulation, namely that users will remain aware of the use of a tool, may start to look unconvincing. Because in this case, the interaction of users with environments that incorporate the rules of how the environment, including the patterns of human behaviours within it, changes over the course of time, may, with prolonged use, also change the users behaviour. This, however, takes us far beyond the scope of the kinds of information and communication tools that are currently emerging.

The mode of interaction and the nature of virtual worlds will thus be a product of the convergence of a number of devices and systems. Yet, as we have seen, even in the case of existing entertainment games, including VR games and simulator games, the mode of interaction depends on the context in which these systems are used. If we then add to this the possibilities of convergence with other information and communication technologies, and of incorporating other forms of computer simulation within VR systems, then it becomes apparent that the current shift among these devices and techniques is subject to a range of intersecting strands of innovation. One way of giving an indication of the profound uncertainties surrounding this shift is by noting that both the interaction with virtual worlds as well as the question of single- versus multi-user virtual worlds have begun to blur the distinction between information technologies (often associated with computers) and communication technologies (devices for the exchange of messages and meanings). In spite - or perhaps because - of such conceptual disarray, virtual worlds are sure to shape the humancomputer interfaces on tomorrow's electronic frontier. 


\section{References}

Biocca, F. (1992). Communication Within Virtual Reality: Creating a Space for Research, Journal of Communication, 42 (4), pp. 5-22.

Collins, R. (1992). Can Sociology Create an Artificial Intelligence? in his Sociological Insight: An Introduction to Non-Obvious Sociology, Oxford: Oxford University Press, pp.155-184.

Curtis, P. and Nichols, D. (1993) MUDs Grow Up: Social Virtual Reality in the Real World, Palo Alto: Xerox PARC.

Fahlen, L. (1993) Virtual Reality and the MultiG Project, in Proceedings of the Third Annual Conference on Virtual Reality London: Meckler, pp.78-86.

Giles, W., Schroeder, R. and Cleal, B. (1994). Virtual Reality and the Future of Interactive Games, in H.-J.Warnecke and H.-J.Bullinger (Eds.) Virtual Reality '94: Anwendungen und Trends, Berlin: Springer, pp.377-391.

Kalawsky, R (1993). The Science of Virtual Reality and Virtual Environments, Reading, MA: Addison Wesley.

Paul, R. and Balmer, D. (1993). Simulation Modelling. Bromley: Chartwell-Bratt.

Salmon, R. and Slater, M. (1987). Computer Graphics: Systems and Concepts, Reading, MA: Addison Wesley.

Schroeder, R (1993). Virtual Reality in the Real World: History, Applications, Projections, in Futures: the Journal of Forecasting, Planning and Policy, pp.963-973.

Schroeder, R. (1994). Worlds in Cyberspace: A Typology of Virtual Realities and their Social Contexts, in Huw Jones, John Vince and Rae Earnshaw (eds) Proceedings of the British Computer Society Conference, Virtual Reality Applications, Leeds.

Slater, M. and Usoh, M. (1993). The Influence of a Virtual Body on presence in Immersive Virtual Environments,' in Proceedings of the Third Annual Conference on Virtual Reality London: Meckler, pp.34-42. 
Sutherland, I. (1965). The Ultimate Display, in Proceedings of International Federation of Information Processing. Congress, pp.506-508.

Waldern, J. (1994). A Note on Software Design of Virtual Team-mates and Virtual Opponents, in Sandra Helsel (ed) Proceedings of the Fourth Annual Conference on Virtual Reality London: Mecklermedia, pp.120-125.

Walker, J. (1990). Through the Looking Glass, in Brenda Laurel (ed) The Art of Human-Computer Interface Design Reading MA: Addison-Wesley, pp.439-447.

White, J.D. (1992) 'SIMCITY' in Simulation \& Gaming, 23 (1), pp.120-123. 
